\title{
Extended Formulations for Independence Polytopes of Regular Matroids
}

\author{
Volker Kaibel • Jon Lee • Matthias \\ Walter • Stefan Weltge
}

Received: date / Accepted: date

\begin{abstract}
We show that the independence polytope of every regular matroid has an extended formulation of size quadratic in the size of its ground set. This generalizes a similar statement for (co-)graphic matroids, which is a simple consequence of Martin's extended formulation for the spanning-tree polytope. In our construction, we make use of Seymour's decomposition theorem for regular matroids. As a consequence, the extended formulations can be computed in polynomial time.
\end{abstract}

Keywords extended formulation - independence polytope · regular matroid . decomposition

Mathematics Subject Classification (2010) 52Bxx

\section{Introduction}

The theory of extended formulations deals with the concept of representing polytopes as linear projections of other polytopes. An extension of a polytope $P$ is some polytope $Q$ together with a linear map $\pi$ such that $\pi(Q)=P$. Given an outer description of $Q$ by means of linear inequalities and equations, this is called an extended formulation for $P$. The size of an extension $(Q, \pi)$ is defined as the number of facets of $Q$. The smallest size of any extension of a polytope

V. Kaibel and S. Weltge acknowledge support by Deutsche Forschungsgemeinschaft (KA 1616/4-1). J. Lee was partially supported by NSF grant CMMI-1160915 and ONR grant N00014-14-1-0315.

V. Kaibel, M. Walter, S. Weltge

Universitätsplatz 2, 39106 Magdeburg, Germany

E-mail: [kaibel,matthias.walter,weltge]@ovgu.de

J. Lee

Department of Industrial and Operations Engineering 1205 Beal Avenue, The University of Michigan, Ann Arbor, MI 48109-2117, USA

E-mail: jonxlee@umich.edu 
$P$ (i.e., the smallest number of inequalities in any extended formulation for $P$ ) is called the extension complexity of $P$ and is denoted by xc $(P)$.

The construction of extended formulations has played an important role in the design of many algorithms solving combinatorial-optimization problems. It turns out that polytopes associated to tractable combinatorial-optimization problems often admit polynomial size (in their dimension) extensions. However, the area of extended formulation has received renewed attraction due to recent results establishing exponential lower bounds on the extension complexities of certain polytopes including the TSP polytope 4 and the perfectmatching polytope [12. While most recent research has focused on improving and extending these results, this paper aims at contributing new positive results pertaining to a well-known class of combinatorial polytopes, namely independence polytopes of regular matroids.

Given a matroid $\mathcal{M}=(E, \mathcal{I})$ with ground set $E$ and independent sets $\mathcal{I}$, the independence polytope of $\mathcal{M}$ is defined as

$$
P(\mathcal{M}):=\operatorname{conv}\{\chi(I) \mid I \in \mathcal{I}\},
$$

where $\chi(I) \in\{0,1\}^{E}$ is the characteristic vector of $I$ with $\chi(I)_{e}=1$ if and only if $e \in I$. Independence polytopes of matroids are central objects in the field of combinatorial optimization. It is well-known that all facet-defining inequalities for $P(\mathcal{M})$ are nonnegativity constraints or inequalities of the form $\sum_{i \in S} x_{i} \leq$ $r(S)$ with $S \subseteq E$, where $r$ denotes the rank function of $\mathcal{M}$. Furthermore, any linear function can be maximized over $P(\mathcal{M})$ by a simple greedy algorithm involving only a linear number of independence oracle calls. For more results on independence polytopes of matroids, see, e.g., Schrijver [14].

In this sense, independence polytopes of matroids are well-understood. One might wonder whether all such polytopes admit polynomial size extended formulations. Unfortunately, this question was answered negatively by Rothvoss [1] who showed that there exists a family of independence polytopes of matroids having extension complexity growing exponentially in the dimension. On the positive side, there are only a few interesting classes of matroids for which we know that the corresponding independence polytopes admit polynomial size extensions. As we will see, using Martin's 9 extended formulation for the spanning-forest polytope, it is easy to derive quadratic size extended formulations for independence polytopes of graphic and cographic matroids. Recently, this has been generalized by Iwata et al. 6] to the class of "sparsity matroids" and later in [1] to the even more general class of "count matroids". In this work, we derive quadratic-size extended formulations for independence polytopes of all regular matroids - another, well-known superclass of (co-)graphic matroids. While the notion of extension complexity only captures existence, the extended formulations presented in this paper indeed can be constructed by a polynomial-time algorithm for regular matroids specified by matrix representations.

Outline In Section 2, we recall known results implying quadratic-size extended formulations for independence polytopes of graphic and cographic matroids. 
Section 3 contains basic definitions and facts about linear and regular matroids including 1-, 2- and 3-sums of regular matroids and Seymour's decomposition theorem. Then, in Section 4, we give an alternative characterization of independent sets in a 1-, 2- or 3-sum. This allows us to derive quadratic-size extended formulations for independence polytopes of regular matroids, as our main result. Finally, we give remarks on applications to related classes of matroids and pose some open questions in Section 5

\section{Graphic and Cographic Matroids}

Classic examples of matroids are graphic matroids. Given an undirected graph $G=$ $(V, E)$, the graphic matroid of $G$ has ground set $E$, where a set of edges is independent if and only if it does not contain a cycle. In other words, a set of edges is independent if it is contained in a spanning forest (i.e., a cycle-free subgraph that has the same connected components as $G)$. Thus, if $\mathcal{M}(G)$ is the graphic matroid of some graph $G$, we have

$$
P(\mathcal{M}(G))=\operatorname{conv}\left\{x \in\{0,1\}^{E} \mid x \leq \chi(F) \text { for some spanning forest } F\right\} .
$$

In order to derive an extended formulation for $P(\mathcal{M}(G))$, we make use of the following simple result concerning the monotonization of $0 / 1$-polytopes. Though it can probably be considered folklore, we include a brief proof, since we are not aware of any appropriate reference.

Lemma 1 For $Y \subseteq\{0,1\}^{n}, P:=\operatorname{conv}(Y)$, and

$$
P^{\downarrow}:=\operatorname{conv}\left\{x \in\{0,1\}^{n} \mid x \leq y \text { for some } y \in Y\right\},
$$

we have

$$
P^{\downarrow}=\left\{x \in \mathbb{R}_{+}^{n} \mid \exists y \in P: x \leq y\right\},
$$

thus $\mathrm{xc}\left(P^{\downarrow}\right) \leq \mathrm{xc}(P)+2 n$.

Proof Let us define $Q:=\left\{x \in \mathbb{R}_{+}^{n} \mid \exists x \in P: y \leq x\right\}$. For every $c \in \mathbb{R}^{n}$, setting $\bar{c}_{i}:=\max \left\{c_{i}, 0\right\}$ for all $i \in[n]$ yields

$$
\begin{aligned}
\max \left\{\langle c, x\rangle \mid x \in P^{\downarrow}\right\} & =\max \left\{\langle c, x\rangle \mid x \in\{0,1\}^{n}, x \leq y \text { for some } y \in Y\right\} \\
& =\max \{\langle\bar{c}, y\rangle \mid y \in Y\} \\
& =\max \{\langle\bar{c}, y\rangle \mid y \in P\} \\
& =\max \{\langle c, x\rangle \mid x \in Q\},
\end{aligned}
$$

and thus $P^{\downarrow}=Q$. The term $2 n$ in the claimed inequality is due to the condition $\mathbb{O} \leq x \leq y$ in the description of $Q$. 
Together with Equation (1), Lemma 1 implies

$$
\mathrm{xc}(P(\mathcal{M}(G))) \leq \mathrm{xc}\left(P_{\text {sp.forests }}(G)\right)+2|E|,
$$

where $P_{\text {sp.forests }}(G)$ is the spanning-forest polytope of $G$, i.e., the convex hull of characteristic vectors of spanning forests of $G$.

Closely related to graphic matroids are cographic matroids, which are the duals of graphic matroids. Given an undirected graph $G=(V, E)$, the cographic matroid of $G$ also has ground set $E$, where now a set of edges is independent if and only if it is contained in the complement of a spanning forest. Thus, if $\mathcal{M}^{*}(G)$ is the cographic matroid of some graph $G$, we obtain

$P\left(\mathcal{M}^{*}(G)\right)=\operatorname{conv}\left\{x \in\{0,1\}^{E} \mid x \leq \mathbb{1}-\chi(F)\right.$ for some spanning forest $\left.F\right\}$, where $\mathbb{1}$ denotes the all-ones vector. Hence, again by Lemma $\mathbb{1}$, this implies

$$
\begin{aligned}
\operatorname{xc}\left(P\left(\mathcal{M}^{*}(G)\right)\right) & \leq \mathrm{xc}\left(\mathbb{1}-P_{\text {sp.forests }}(G)\right)+2|E| \\
& =\operatorname{xc}\left(P_{\text {sp.forests }}(G)\right)+2|E| .
\end{aligned}
$$

As Martin 9] showed, the spanning-forest polytope of a graph $G=(V, E)$ admits an extended formulation of size $\mathcal{O}(|V| \cdot|E|)$. Because the spanningforest polytope of a graph is the Cartesian product of the spanning-forest polytopes of its connected components, and because one has $|V| \leq|E|-1$ for every connected graph $G=(V, E)$, we obtain the estimate xc $\left(P_{\text {sp.forests }}(G)\right) \leq$ $\mathcal{O}\left(|E|^{2}\right)$. Using Inequality (2) and Inequality (3), we conclude:

Proposition 1 For any graphic or cographic matroid $\mathcal{M}$ on ground set $E$ we have

$$
\mathrm{xc}(P(\mathcal{M})) \leq \mathcal{O}\left(|E|^{2}\right) .
$$

Moreover, a result by Williams 17 even provides linear-size extended formulations for spanning-forest polytopes in the case of planar graphs. Thus, if $G$ is a planar graph, we can improve the bound in Proposition 1 to $\mathrm{xc}(P(\mathcal{M})) \leq$ $\mathcal{O}(|E|)$

\section{Regular Matroids}

A much more general family of matroids comprising graphic and cographic matroids is the class of linear matroids. Given a matrix $A \in \mathbb{F}^{p \times q}$ with entries in some field $\mathbb{F}$, we denote by

$$
\mathcal{M}_{\mathbb{F}}(A):=\left\{I \subseteq[p+q] \mid(\mathbb{I} A)_{\star, I} \text { has full column-rank over } \mathbb{F}\right\}
$$

the (set of independent sets of the) matroid defined by $A$, where $\mathbb{I}$ is the $p \times p$ identity matrix and $(\mathbb{I} A)_{\star, I}$ denotes the submatrix of $(\mathbb{I} A)$ consisting of all rows but only the columns $I$. Therefore, the cardinality of the ground set of $\mathcal{M}_{\mathbb{F}}(A)$ is $p+q$, i.e., the number columns of the identity-extension $(\mathbb{I} A)$ of the matrix $A$. Note that this use of notation differs, e.g., from Oxley [10, but 
is in accordance to Schrijver's book [13, Chap. 19]. Here, we allow $A$ to have $q=0$ columns (in which case $\mathcal{M}_{\mathbb{F}}(A)$ is a free matroid on $p$ elements with all subsets being independent) or $p=0$ rows (in which case $\mathcal{M}_{\mathbb{F}}(A)$ is a matroid on $q$ elements such that the empty set is the only independent set), but we will always have $p+q>0$. If a matroid $\mathcal{M}$ is isomorphic to $\mathcal{M}_{\mathbb{F}}(A)$ for some matrix $A$ and some field $\mathbb{F}$, we say that $\mathcal{M}$ can be represented (by $A$ ) over $\mathbb{F}$. The class of linear matroids consists of all matroids that can be represented over some field.

In this paper, we focus on the well-known class of matroids that can be represented over every field, namely regular matroids. It can be shown that a matroid is regular if and only if it can be represented by a totally-unimodular matrix over $\mathbb{R}$ (see, e.g., [13, Chap. 19]). Note that for a totally-unimodular matrix $A$, the matroid $\mathcal{M}_{\mathbb{F}}(A)$ does not depend on the specific choice of the field $\mathbb{F}$. Thus, we will mainly work over the most simple field $\mathbb{F}_{2}$, with two elements.

Key examples of regular matroids are graphic matroids. Let $G=(V, E)$ be an undirected connected graph. Choosing some $T \subseteq E$ that forms a spanning tree of $G$ and assigning some orientation to all edges in $E$, let us construct a matrix $A \in\{0,1,-1\}^{T \times E}$ as follows: For every pair of (directed) edges $t \in T$ and $e=(v, w) \in E$, set the entry $A_{t, e}$ to 1 or -1 if the path from $v$ to $w$ in $T$ passes through $t$ in forward or backward direction, respectively, and to 0 if it does not pass through $t$ at all. It can be shown that $\mathcal{M}(G)$ is (isomorphic to) $\mathcal{M}_{\mathbb{R}}(A)$ and that $A$ is totally unimodular. In particular, this implies that $\mathcal{M}(G)$ is regular.

Not every regular matroid is graphic or cographic. However, it turns out that all remaining regular matroids can be constructed from only graphic matroids, cographic matroids and matroids of size at most ten.

\subsection{Seymour's Decomposition Theorem}

From its basic definition, there seems to be no hint concerning crucial properties of regular matroids to exploit in order to obtain polynomial-size extended formulations for the corresponding independence polytopes. Fortunately, it turns out that Seymour's celebrated decomposition theorem provides suitable access for our purpose. In order to state the result, we need to define a few operations on regular matroids. Since we aim at describing how to actually construct extended formulations for regular matroids given by representing matrices, we prefer to make use of the decomposition in terms of matrices (as you can find it, e.g., in Schrijver's book [13]) rather than in terms of purely structural matroid theory (as, e.g., in Oxley's book [10]). In what follows, all matrices and operations are considered over $\mathbb{F}_{2}$. For convenience, we will therefore write $\mathcal{M}(A):=\mathcal{M}_{\mathbb{F}_{2}}(A)$ for any 0/1-matrix $A$.

Let $\mathcal{M}, \mathcal{M}_{1}$ and $\mathcal{M}_{2}$ be binary matroids, i.e., matroids represented over $\mathbb{F}_{2}$. We say that $\mathcal{M}$ is a 1 -sum of $\mathcal{M}_{1}$ and $\mathcal{M}_{2}$ if there exist matrices $A, B$ 
such that

$$
\mathcal{M}_{1}=\mathcal{M}(A), \quad \mathcal{M}_{2}=\mathcal{M}(B), \quad \mathcal{M}=\mathcal{M}\left(\begin{array}{ll}
A & \mathbb{O} \\
\mathbb{O} & B
\end{array}\right)
$$

holds. We say that $\mathcal{M}$ is a 2 -sum of $\mathcal{M}_{1}$ and $\mathcal{M}_{2}$ if there exist matrices $A, B$ and column vectors $a, b$ such that

$$
\mathcal{M}_{1}=\mathcal{M}(a A), \quad \mathcal{M}_{2}=\mathcal{M}\left(\begin{array}{l}
b^{\top} \\
B
\end{array}\right), \quad \mathcal{M}=\mathcal{M}\left(\begin{array}{cc}
A & a b^{\top} \\
\mathbb{O} & B
\end{array}\right)
$$

holds. Finally, we say that $\mathcal{M}$ is a 3-sum of $\mathcal{M}_{1}$ and $\mathcal{M}_{2}$ if there exist matrices $A, B$ and column vectors $a, b, c, d$ such that

$$
\mathcal{M}_{1}=\mathcal{M}\left(\begin{array}{lll}
a & a & A \\
0 & 1 & c^{\top}
\end{array}\right), \quad \mathcal{M}_{2}=\mathcal{M}\left(\begin{array}{lll}
0 & 1 & b^{\top} \\
d & d & B
\end{array}\right), \quad \mathcal{M}=\mathcal{M}\left(\begin{array}{cc}
A & a b^{\top} \\
d c^{\top} & B
\end{array}\right)
$$

holds. In each of the above definitions, we allow $A$ to consist of no columns and $B$ to consist of no rows. Seymour's characterization of regular matroids yields the following:

Theorem 1 (Regular-Matroid Decomposition Theorem [15], see [13, Thm. 19.6]) For every regular matroid $\mathcal{M}$ there exists a rooted binary tree $T$ whose nodes are binary matroids such that

- the root of $T$ is $\mathcal{M}$,

- each non-leaf node of $T$ is isomorphic to a $k$-sum of its two children for some $k \in\{1,2,3\}$,

- each leaf of $T$ is a graphic matroid, a cographic matroid or has size at most ten.

- each leaf of $T$ has a ground set of cardinality at least three, and

- whenever a non-leaf node of $T$ is isomorphic to a 3-sum of its children, both children have ground sets of cardinality at least seven.

Moreover, for input matrix $A \in \mathbb{F}_{2}^{m \times n}$ such a decomposition of $\mathcal{M}=\mathcal{M}(A)$ can be computed in time polynomially bounded in $m$ and $n$.

In Schrijver's book [13, the above statement is formulated in terms of a decomposition theorem for totally-unimodular matrices, for which he allows certain additional operations on matrices. Those are pivoting, permutations of rows or columns as well as scaling of rows or columns by -1 , which all do not change the isomorphism type of the matroid. Furthermore, there are the operations of adding an all-zero row or column, adding a unit-vector as a row or column, or repeating a row or column. It can be easily seen that these ones can be obtained (up to isomorphism) as 2-sums with certain matroids on ground sets of cardinality three.

Finally, Schrijver uses the transposition of a matrix as a particular operation, which, in the strict sense, may be performed at any node in the decomposition tree. Technically, this yields a decomposition tree $T$ in which every 
non-leaf node $\mathcal{M}$ is a $k$-sum of its two children, or it has only one child $\mathcal{M}^{\prime}$ with $\mathcal{M}=\mathcal{M}(A)$ and $\mathcal{M}^{\prime}=\mathcal{M}\left(A^{\top}\right)$ for some matrix $A \in \mathbb{F}_{2}^{m \times n}$. Let us argue that we can modify $T$ such that no transposition has to be performed at all. Let $\mathcal{M}$ be a node that has only one child $\mathcal{M}^{\prime}$. If $\mathcal{M}^{\prime}$ also has exactly one child $\mathcal{M}^{\prime \prime}$, then $\mathcal{M}=\mathcal{M}^{\prime \prime}$ and we can shorten the tree. If $\mathcal{M}^{\prime}$ has two children, it is a $k$-sum. It is easy to see that also $\mathcal{M}$ is a $k$-sum of two regular matroids $\mathcal{M}_{1}$ and $\mathcal{M}_{2}$ on ground sets of corresponding sizes. Since $\mathcal{M}_{1}$ and $\mathcal{M}_{2}$ are regular matroids, there exist decomposition trees (that still may include transpositions) $T_{1}$ and $T_{2}$ with $\mathcal{M}_{1}$ and $\mathcal{M}_{2}$ as roots, respectively. In this case, we delete the subtree starting at $\mathcal{M}^{\prime}$ and connect $\mathcal{M}$ with $T_{1}$ and $T_{2}$. Using this procedure, we end up with a decomposition tree for which all nodes $\mathcal{M}$ with only child $\mathcal{M}^{\prime}$ satisfy that $\mathcal{M}^{\prime}$ is a leaf node. Since the transposition of a matrix corresponds to taking the dual of the induced matroid, we can remove all such leaf nodes and obtain a decomposition tree as in Theorem 1 .

\section{Main Result}

As described in Section 2, we already have small extended formulations for the independence polytopes of the leaf nodes in decompositions as in Theorem 1 . (Note that the leaf nodes that are not graphic or cographic have size bounded by some constant.) Given a decomposition tree, we are going to construct an extended formulation for the independence polytope of the root node whose size is small in terms of the sum of the sizes of the extended formulations of the independence polytopes of the leaf nodes. In order to deduce our main result from such a construction later, we first bound the sizes of components in decomposition trees.

Lemma 2 Let $\mathcal{M}$ be a regular matroid on ground set $E$, and let $T$ be a decomposition tree of $\mathcal{M}$ as in Theorem 1. Then the sum of the cardinalities of the ground sets of leaf nodes of $T$ can be bounded linearly in $|E|$.

Proof Let $f(n)$ denote the largest sum of cardinalities of the ground sets of leaf nodes in any decomposition tree as in Theorem 1 for a regular matroid whose ground set has cardinality $n$. Defining

$$
\begin{aligned}
g(n):=\max (\{n\} & \cup\{g(t)+g(n-t) \mid 3 \leq t \leq n-3\} \\
& \cup\{g(t+1)+g(n-t+1) \mid 2 \leq t \leq n-2\} \\
& \cup\{g(t+3)+g(n-t+3) \mid 4 \leq t \leq n-4\})
\end{aligned}
$$

for all $n \geq 2$, and setting $g(1):=1$, we read off from Theorem 1 that we have $f(n) \leq g(n)$ for all $n \geq 1$ (note that whenever a node with ground set of size $n$ is the 1-, 2-, or 3-sum of its two children with ground sets of sizes $n_{1}$ and $n_{2}$, then $n_{1}+n_{2}$ equals $n$ minus 0,2 , or 6 , respectively). Inspecting the function $g$ more closely, we find that we have $g(7)=15, g(8)=30$ and

$$
g(n)=\max (\{g(t+3)+g(n-t+3) \mid 4 \leq t \leq n-4\}),
$$


for all $n \geq 9$. From this one deduces $g(n)=15(n-6)$ for all $n \geq 7$ by induction.

Next, for our construction it is necessary to characterize the independent sets of $k$-sums as defined above. For the sake of completeness, we include a full proof of the following lemma in matrix language instead of deriving the statements from known results in structural matroid theory. We use the symbol $\uplus$ in order to emphasize when a union is taken of two sets with empty intersection.

Lemma 3 Let $\mathcal{M}=(E, \mathcal{I}), \mathcal{M}_{1}=\left(E_{1}, \mathcal{I}_{1}\right)$, and $\mathcal{M}_{2}=\left(E_{2}, \mathcal{I}_{2}\right)$ be binary matroids with $E_{1} \cap E_{2}=\emptyset$ such that $\mathcal{M}$ is a $k$-sum of $\mathcal{M}_{1}$ and $\mathcal{M}_{2}$. Then the independent sets of $\mathcal{M}$ can be characterized (up to isomorphism) as follows:

$-k=1: \mathcal{I}=\left\{I_{1} \uplus I_{2} \mid I_{1} \in \mathcal{I}_{1}, I_{2} \in \mathcal{I}_{2}\right\}$

- $k=2: \mathcal{M}$ is a 1-sum of a minor of $\mathcal{M}_{1}$ and a minor of $\mathcal{M}_{2}$; or there exist elements $r_{1} \in E_{1}, r_{2} \in E_{2}$ satisfying

$$
\begin{aligned}
\mathcal{I}=\left\{\left(I_{1} \backslash\left\{r_{1}\right\}\right) \uplus\left(I_{2} \backslash\left\{r_{2}\right\}\right):\right. & I_{1} \in \mathcal{I}_{1}, I_{2} \in \mathcal{I}_{2}, \\
& \left.\left|I_{1} \cap\left\{r_{1}\right\}\right|+\left|I_{2} \cap\left\{r_{2}\right\}\right|=1\right\} .
\end{aligned}
$$

- $k=3: \mathcal{M}$ is a 2-sum of a minor of $\mathcal{M}_{1}$ and a minor of $\mathcal{M}_{2}$; or there exist pairwise distinct elements $r_{1}, p_{1}, q_{1} \in E_{1}, r_{2}, p_{2}, q_{2} \in E_{2}$ satisfying

$$
\begin{aligned}
\mathcal{I}=\left\{\left(I_{1} \backslash\left\{r_{1}, p_{1}, q_{1}\right\}\right) \uplus\left(I_{2} \backslash\left\{r_{2}, p_{2}, q_{2}\right\}\right):\right. & I_{1} \in \mathcal{I}_{1}, I_{2} \in \mathcal{I}_{2}, \\
& \left|I_{1} \cap\left\{r_{1}\right\}\right|+\left|I_{2} \cap\left\{r_{2}\right\}\right|=1, \\
& \left|I_{1} \cap\left\{p_{1}\right\}\right|+\left|I_{2} \cap\left\{p_{2}\right\}\right|=1, \\
& \left.\left|I_{1} \cap\left\{q_{1}\right\}\right|+\left|I_{2} \cap\left\{q_{2}\right\}\right|=1\right\} .
\end{aligned}
$$

Proof Note that the statement for the case $k=1$ follows trivially from the definition of a 1-sum. Let us consider the case $k=2$ and suppose that we have $\mathcal{M}_{1}=\mathcal{M}\left(\begin{array}{ll}a & A\end{array}\right), \mathcal{M}_{2}=\mathcal{M}\left(\begin{array}{c}b^{\top} \\ B\end{array}\right)$ and $\mathcal{M}=\mathcal{M}\left(\begin{array}{cc}A & a b^{\top} \\ \mathbb{O} & B\end{array}\right)$. The identity-extension of $\left(\begin{array}{cc}A & a b^{\top} \\ \mathbb{O} & B\end{array}\right)$ (after permuting columns) is

$$
\left(\begin{array}{cccc}
\mathbb{I} & A & \mathbb{O} & a b^{\top} \\
\mathbb{O} & \mathbb{O} & \mathbb{I} & B
\end{array}\right) .
$$

Denote the elements corresponding to the first column of ( $a A$ ) and the first column of the identity-extension of $\left(\begin{array}{c}b^{\top} \\ B\end{array}\right)$ (being the first unit vector) by $r_{1}$ and $r_{2}$, respectively. With this notation, we may assume that we have $E=$ $\left(E_{1} \backslash\left\{r_{1}\right\}\right) \uplus\left(E_{2} \backslash\left\{r_{2}\right\}\right)$. In addition, note that if $a=\mathbb{O}$ holds, then $\mathcal{M}$ is a 1-sum of $\mathcal{M}(A)$ and $\mathcal{M}(B)$, which are minors of $\mathcal{M}_{1}$ and $\mathcal{M}_{2}$, respectively. Thus, we may further assume that $a \neq \mathbb{O}$ holds and obtain that a subset of $E$ is independent (in $\mathcal{M}$ ) if and only if it is of the form $J_{1} \uplus J_{2}$ with $J_{1}=I_{1} \backslash\left\{r_{1}\right\}$ and $J_{2}=I_{2} \backslash\left\{r_{2}\right\}$ where $I_{1} \in \mathcal{I}_{1}$ and (due to $a \neq \mathbb{O}$ ) $I_{2} \in \mathcal{I}_{2}$ such that

$$
\operatorname{span}_{J_{1}}\left(\begin{array}{ll}
\mathbb{I} & A \\
\mathbb{O} & \mathbb{O}
\end{array}\right) \cap \operatorname{span}_{J_{2}}\left(\begin{array}{cc}
\mathbb{O} & a b^{\top} \\
\mathbb{I} & B
\end{array}\right)=\{\mathbb{O}\}
$$


holds, where $\operatorname{span}_{J}(\cdot)$ denotes the $\mathbb{F}_{2}$-subspace spanned by the columns corresponding to $J$. Because we have (with $\operatorname{span}(\cdot)$ denoting the $\mathbb{F}_{2}$-subspace spanned by all columns)

$$
\operatorname{span}\left(\begin{array}{cc}
\mathbb{I} & A \\
\mathbb{O} & \mathbb{O}
\end{array}\right) \cap \operatorname{span}\left(\begin{array}{cc}
\mathbb{O} & a b^{\top} \\
\mathbb{I} & B
\end{array}\right) \backslash\{\mathbb{O}\} \subseteq\left\{\left(\begin{array}{l}
a \\
\mathbb{O}
\end{array}\right)\right\},
$$

the latter condition is equivalent to

$$
\left(\begin{array}{l}
a \\
\mathbb{O}
\end{array}\right) \notin \operatorname{span}_{J_{1}}\left(\begin{array}{ll}
\mathbb{I} & A \\
\mathbb{O} & \mathbb{O}
\end{array}\right) \quad \text { or } \quad\left(\begin{array}{l}
a \\
\mathbb{O}
\end{array}\right) \notin \operatorname{span}_{J_{2}}\left(\begin{array}{cc}
\mathbb{O} & a b^{\top} \\
\mathbb{I} & B
\end{array}\right)
$$

which is equivalent to (recall $a \neq \mathbb{O}$ )

$$
a \notin \operatorname{span}_{J_{1}}(\mathbb{I} A) \quad \text { or } \quad\left(\begin{array}{l}
1 \\
\mathbb{O}
\end{array}\right) \notin \operatorname{span}_{J_{2}}\left(\begin{array}{cc}
\mathbb{O}^{\top} & b^{\top} \\
\mathbb{I} & B
\end{array}\right),
$$

and thus to

$$
J_{1} \cup\left\{r_{1}\right\} \in \mathcal{I}_{1} \quad \text { or } \quad J_{2} \cup\left\{r_{2}\right\} \in \mathcal{I}_{2} .
$$

Hence, we obtain

$$
\begin{aligned}
\mathcal{I}=\left\{\left(I_{1} \backslash\left\{r_{1}\right\}\right) \uplus\left(I_{2} \backslash\left\{r_{2}\right\}\right):\right. & I_{1} \in \mathcal{I}_{1}, I_{2} \in \mathcal{I}_{2}, \\
& \left.\left|I_{1} \cap\left\{r_{1}\right\}\right|+\left|I_{2} \cap\left\{r_{2}\right\}\right| \geq 1\right\} \\
=\left\{\left(I_{1} \backslash\left\{r_{1}\right\}\right) \uplus\left(I_{2} \backslash\left\{r_{2}\right\}\right):\right. & I_{1} \in \mathcal{I}_{1}, I_{2} \in \mathcal{I}_{2}, \\
& \left.\left|I_{1} \cap\left\{r_{1}\right\}\right|+\left|I_{2} \cap\left\{r_{2}\right\}\right|=1\right\},
\end{aligned}
$$

where the last equality follows from the fact that

$$
\left(E_{1} \uplus E_{2},\left\{I_{1} \uplus I_{2} \mid I_{1} \in \mathcal{I}_{1}, I_{2} \in \mathcal{I}_{2}\right\}\right)
$$

is an independence system (in fact, a matroid that is the direct sum of matroids).

For the remaining case $k=3$, let $\mathcal{M}_{1}=\mathcal{M}\left(\begin{array}{lll}a & a & A \\ 0 & 1 & c^{\top}\end{array}\right), \mathcal{M}_{2}=\mathcal{M}\left(\begin{array}{lll}0 & 1 & b^{\top} \\ d & d & B\end{array}\right)$ and $\mathcal{M}=\mathcal{M}\left(\begin{array}{cc}A & a b^{\top} \\ d c^{\top} & B\end{array}\right)$. The identity extension of $\left(\begin{array}{cc}A & a b^{\top} \\ d c^{\top} & B\end{array}\right)$ (after permuting columns) is

$$
\left(\begin{array}{cccc}
\mathbb{I} & A & \mathbb{O} & a b^{\top} \\
\mathbb{O} & d c^{\top} & \mathbb{I} & B
\end{array}\right)
$$

Let us denote certain elements corresponding to the columns of the identity extensions of $\left(\begin{array}{ccc}a & a & A \\ 0 & 1 & c^{\top}\end{array}\right)$ and $\left(\begin{array}{lll}0 & 1 & b^{\top} \\ d & d & B\end{array}\right)$, respectively, as follows:

$$
\begin{aligned}
& \left(\begin{array}{lllll}
1 & \mathbb{O} & a & a & A \\
\mathbb{O} & \mathbb{I} & 0 & 1 & c^{\top}
\end{array}\right) \quad\left(\begin{array}{lllll}
1 & \mathbb{O} & 0 & 1 & b^{\top} \\
\mathbb{O} & \mathbb{I} & d & d & B
\end{array}\right) \\
& \begin{array}{ccccc}
\uparrow & \uparrow & \uparrow & \uparrow & \uparrow \\
p_{1} & r_{1} & q_{1} & r_{2} & p_{2} q_{2}
\end{array}
\end{aligned}
$$


With this notation, we may assume that we have $E=\left(E_{1} \backslash\left\{r_{1}, p_{1}, q_{1}\right\}\right) \uplus$ $\left(E_{2} \backslash\left\{r_{2}, p_{2}, q_{2}\right\}\right)$. In addition, note that if $d=\mathbb{O}$ holds, then $\mathcal{M}$ is a 2-sum of $\mathcal{M}\left(\begin{array}{ll}a & A\end{array}\right)$ and $\mathcal{M}\left(\begin{array}{c}b^{\top} \\ B\end{array}\right)$, which are minors of $\mathcal{M}_{1}$ and $\mathcal{M}_{2}$, respectively. A similar argument holds for the case $a=\mathbb{O}$. Thus, we may further assume that $d \neq \mathbb{O}$ and $a \neq \mathbb{O}$ holds. In this case, a subset of $E$ is independent (in $\mathcal{M}$ ) if and only if it is of the form $J_{1} \uplus J_{2}$ with $J_{1}=I_{1} \backslash\left\{r_{1}, p_{1}, q_{1}\right\}$ and $J_{2}=I_{2} \backslash\left\{r_{2}, p_{2}, q_{2}\right\}$ where (due to $d \neq \mathbb{O}$ ) $I_{1} \in \mathcal{I}_{1}$ and (due to $a \neq \mathbb{O}$ ) $I_{2} \in \mathcal{I}_{2}$ such that

$$
\operatorname{span}_{J_{1}}\left(\begin{array}{cc}
\mathbb{I} & A \\
\mathbb{O} & d c^{\top}
\end{array}\right) \cap \operatorname{span}_{J_{2}}\left(\begin{array}{cc}
\mathbb{O} & a b^{\boldsymbol{\top}} \\
\mathbb{I} & B
\end{array}\right)=\{\mathbb{O}\}
$$

holds. Because we have

$$
\operatorname{span}\left(\begin{array}{cc}
\mathbb{I} & A \\
\mathbb{O} & d c^{\top}
\end{array}\right) \cap \operatorname{span}\left(\begin{array}{cc}
\mathbb{O} & a b^{\boldsymbol{\top}} \\
\mathbb{I} & B
\end{array}\right) \backslash\{\mathbb{O}\} \subseteq\left\{\left(\begin{array}{l}
a \\
\mathbb{O}
\end{array}\right),\left(\begin{array}{l}
\mathbb{O} \\
d
\end{array}\right),\left(\begin{array}{l}
a \\
d
\end{array}\right)\right\}
$$

the latter condition is equivalent to

$$
\begin{aligned}
& {\left[\left(\begin{array}{l}
a \\
\mathbb{O}
\end{array}\right) \notin \operatorname{span}_{J_{1}}\left(\begin{array}{cc}
\mathbb{I} & A \\
\mathbb{O} & d c^{\top}
\end{array}\right) \quad \text { or } \quad\left(\begin{array}{l}
a \\
\mathbb{O}
\end{array}\right) \notin \operatorname{span}_{J_{2}}\left(\begin{array}{cc}
\mathbb{O} & a b^{\top} \\
\mathbb{I} & B
\end{array}\right)\right] \quad \text { and }} \\
& {\left[\left(\begin{array}{l}
\mathbb{O} \\
d
\end{array}\right) \notin \operatorname{span}_{J_{1}}\left(\begin{array}{cc}
\mathbb{I} & A \\
\mathbb{O} & d c^{\top}
\end{array}\right) \quad \text { or } \quad\left(\begin{array}{l}
\mathbb{O} \\
d
\end{array}\right) \notin \operatorname{span}_{J_{2}}\left(\begin{array}{cc}
\mathbb{O} & a b^{\top} \\
\mathbb{I} & B
\end{array}\right)\right] \quad \text { and }} \\
& {\left[\left(\begin{array}{l}
a \\
d
\end{array}\right) \notin \operatorname{span}_{J_{1}}\left(\begin{array}{cc}
\mathbb{I} & A \\
\mathbb{O} & d c^{\top}
\end{array}\right) \quad \text { or } \quad\left(\begin{array}{l}
a \\
d
\end{array}\right) \notin \operatorname{span}_{J_{2}}\left(\begin{array}{cc}
\mathbb{O} & a b^{\top} \\
\mathbb{I} & B
\end{array}\right)\right] \text {, }}
\end{aligned}
$$

which due to $a \neq \mathbb{O}$ and $d \neq \mathbb{O}$ is equivalent to

$$
\begin{aligned}
& {\left[\left(\begin{array}{l}
a \\
0
\end{array}\right) \notin \operatorname{span}_{J_{1}}\left(\begin{array}{cc}
\mathbb{I} & A \\
\mathbb{O}^{\top} & c^{\top}
\end{array}\right) \quad \text { or } \quad\left(\begin{array}{l}
1 \\
\mathbb{O}
\end{array}\right) \notin \operatorname{span}_{J_{2}}\left(\begin{array}{cc}
\mathbb{O}^{\top} & b^{\top} \\
\mathbb{I} & B
\end{array}\right)\right] \quad \text { and }} \\
& {\left[\left(\begin{array}{l}
\mathbb{O} \\
1
\end{array}\right) \notin \operatorname{span}_{J_{1}}\left(\begin{array}{cc}
\mathbb{I} & A \\
\mathbb{O}^{\top} & c^{\top}
\end{array}\right) \quad \text { or } \quad\left(\begin{array}{l}
0 \\
d
\end{array}\right) \notin \operatorname{span}_{J_{2}}\left(\begin{array}{cc}
\mathbb{O}^{\top} & b^{\top} \\
\mathbb{I} & B
\end{array}\right)\right] \quad \text { and }} \\
& {\left[\left(\begin{array}{l}
a \\
1
\end{array}\right) \notin \operatorname{span}_{J_{1}}\left(\begin{array}{cc}
\mathbb{I} & A \\
\mathbb{O}^{\top} & c^{\top}
\end{array}\right) \quad \text { or } \quad\left(\begin{array}{l}
1 \\
d
\end{array}\right) \notin \operatorname{span}_{J_{2}}\left(\begin{array}{cc}
\mathbb{O}^{\top} & b^{\top} \\
\mathbb{I} & B
\end{array}\right)\right] \text {, }}
\end{aligned}
$$

and thus to

$$
\begin{aligned}
& {\left[J_{1} \cup\left\{r_{1}\right\} \in \mathcal{I}_{1} \quad \text { or } \quad J_{2} \cup\left\{r_{2}\right\} \in \mathcal{I}_{2}\right] \quad \text { and }} \\
& {\left[J_{1} \cup\left\{p_{1}\right\} \in \mathcal{I}_{1} \quad \text { or } \quad J_{2} \cup\left\{p_{2}\right\} \in \mathcal{I}_{2}\right] \quad \text { and }} \\
& {\left[J_{1} \cup\left\{q_{1}\right\} \in \mathcal{I}_{1} \quad \text { or } \quad J_{2} \cup\left\{q_{2}\right\} \in \mathcal{I}_{2}\right] \text {. }}
\end{aligned}
$$


Hence, we obtain

$$
\begin{aligned}
\mathcal{I}=\left\{\left(I_{1} \backslash\left\{r_{1}, p_{1}, q_{1}\right\}\right) \uplus\left(I_{2} \backslash\left\{r_{2}, p_{2}, q_{2}\right\}\right):\right. & I_{1} \in \mathcal{I}_{1}, I_{2} \in \mathcal{I}_{2}, \\
& \left|I_{1} \cap\left\{r_{1}\right\}\right|+\left|I_{2} \cap\left\{r_{2}\right\}\right| \geq 1, \\
& \left|I_{1} \cap\left\{p_{1}\right\}\right|+\left|I_{2} \cap\left\{p_{2}\right\}\right| \geq 1, \\
& \left.\left|I_{1} \cap\left\{q_{1}\right\}\right|+\left|I_{2} \cap\left\{q_{2}\right\}\right| \geq 1\right\} \\
=\left\{\left(I_{1} \backslash\left\{r_{1}, p_{1}, q_{1}\right\}\right) \uplus\left(I_{2} \backslash\left\{r_{2}, p_{2}, q_{2}\right\}\right):\right. & I_{1} \in \mathcal{I}_{1}, I_{2} \in \mathcal{I}_{2}, \\
& \left|I_{1} \cap\left\{r_{1}\right\}\right|+\left|I_{2} \cap\left\{r_{2}\right\}\right|=1, \\
& \left|I_{1} \cap\left\{p_{1}\right\}\right|+\left|I_{2} \cap\left\{p_{2}\right\}\right|=1, \\
& \left.\left|I_{1} \cap\left\{q_{1}\right\}\right|+\left|I_{2} \cap\left\{q_{2}\right\}\right|=1\right\},
\end{aligned}
$$

where the last equality again follows from the fact that

$$
\left(E_{1} \uplus E_{2},\left\{I_{1} \uplus I_{2} \mid I_{1} \in \mathcal{I}_{1}, I_{2} \in \mathcal{I}_{2}\right\}\right)
$$

is an independence system.

Finally, we bound the extension complexities of independence polytopes of $k$-sums in terms of their summands.

Lemma 4 Let $\mathcal{M}$ be a $k$-sum of $\mathcal{M}_{1}$ and $\mathcal{M}_{2}$ for some $k \in\{1,2,3\}$. Then we have

$$
\left.\mathrm{xc}(P(\mathcal{M})) \leq \mathrm{xc}\left(P\left(\mathcal{M}_{1}\right)\right)+\operatorname{xc}\left(P\left(\mathcal{M}_{2}\right)\right)\right) .
$$

Proof Let $\mathcal{M}=(E, \mathcal{I})$ be a $k$-sum of $\mathcal{M}_{1}=\left(E_{1}, \mathcal{I}_{1}\right)$ and $\mathcal{M}_{2}=\left(E_{2}, \mathcal{I}_{2}\right)$ (with $E_{1} \cap E_{2}=\varnothing$ ). First, observe that if some matroid $\mathcal{M}^{\prime \prime}$ is a minor of $\mathcal{M}^{\prime}$, then $P\left(\mathcal{M}^{\prime \prime}\right)$ can be obtained by intersecting $P\left(\mathcal{M}^{\prime}\right)$ with a face of the $0 / 1$-cube. Hence, $P\left(\mathcal{M}^{\prime \prime}\right)$ is a coordinate projection of a face of $P\left(\mathcal{M}^{\prime}\right)$ and therefore $\mathrm{xc}\left(P\left(\mathcal{M}^{\prime \prime}\right)\right) \leq \mathrm{xc}\left(P\left(\mathcal{M}^{\prime}\right)\right)$. Thus, by Lemma 3, it remains to consider the case in which there exist pairwise distinct elements $e_{1}, \ldots, e_{t} \in E_{1}$ and pairwise distinct elements $f_{1}, \ldots, f_{t} \in E_{2}$ such that

$$
E=\left(E_{1} \backslash\left\{e_{1}, \ldots, e_{t}\right\}\right) \uplus\left(E_{2} \backslash\left\{f_{1}, \ldots, f_{t}\right\}\right)
$$

and

$$
\begin{aligned}
\mathcal{I}=\left\{\left(I_{1} \backslash\left\{e_{1}, \ldots, e_{t}\right\}\right)\right. & \uplus\left(I_{2} \backslash\left\{f_{1}, \ldots, f_{t}\right\}\right): \\
& I_{1} \in \mathcal{I}_{1}, I_{2} \in \mathcal{I}_{2}, \\
& \left|I_{1} \cap\left\{e_{i}\right\}\right|+\left|I_{2} \cap\left\{f_{i}\right\}\right|=1 \\
& \text { for all } i \in\{1, \ldots, t\}\}
\end{aligned}
$$

holds. Thus, setting

$$
Q:=\left\{(x, y) \in[0,1]^{E_{1}} \times[0,1]^{E_{2}} \mid x_{e_{i}}+y_{f_{i}}=1 \forall i=1, \ldots, t\right\},
$$


we obtain that $P(\mathcal{M})$ is a coordinate projection of

$$
\begin{aligned}
\operatorname{conv}( & \left.\left(P\left(\mathcal{M}_{1}\right) \times P\left(\mathcal{M}_{2}\right)\right) \cap Q \cap\left(\{0,1\}^{E_{1}} \times\{0,1\}^{E_{2}}\right)\right) \\
& =\left(P\left(\mathcal{M}_{1}\right) \times P\left(\mathcal{M}_{2}\right)\right) \cap Q
\end{aligned}
$$

where the equality follows from Edmonds' intersection theorem for matroid polytopes [2] and the fact that $P\left(\mathcal{M}_{1}\right) \times P\left(\mathcal{M}_{2}\right)$ and $Q$ are (faces of) matroid polytopes. In particular, we established

$$
\begin{aligned}
\operatorname{xc}(P(\mathcal{M})) & \left.\leq \operatorname{xc}\left(P\left(\mathcal{M}_{1}\right) \times P\left(\mathcal{M}_{2}\right)\right) \cap Q\right) \\
& =\operatorname{xc}\left(\left\{(x, y) \in P\left(\mathcal{M}_{1}\right) \times P\left(\mathcal{M}_{2}\right) \mid x_{e_{i}}+y_{f_{i}}=1 \forall i=1, \ldots, t\right\}\right) \\
& \leq \operatorname{xc}\left(P\left(\mathcal{M}_{1}\right) \times P\left(\mathcal{M}_{2}\right)\right) \\
& \leq \operatorname{xc}\left(P\left(\mathcal{M}_{1}\right)\right)+\operatorname{xc}\left(P\left(\mathcal{M}_{2}\right)\right) .
\end{aligned}
$$

We remark that [5, Lemma 3.4] gives a similar result on the structure of independence polytopes of matroids arising from 2 -sums. We are now ready to prove our main result.

Theorem 2 For any regular matroid $\mathcal{M}$ on ground set $E$, we have

$$
\mathrm{xc}(P(\mathcal{M})) \leq \mathcal{O}\left(|E|^{2}\right) .
$$

Proof Let $\mathcal{M}_{1}=\left(E_{1}, \mathcal{I}_{1}\right), \ldots, \mathcal{M}_{k}=\left(E_{k}, \mathcal{I}_{k}\right)$ be the leaf nodes in some decomposition tree as in Theorem 1 By Lemma 4, we have that

$$
\operatorname{xc}(P(\mathcal{M})) \leq \sum_{i=1}^{k} \operatorname{xc}\left(P\left(\mathcal{M}_{i}\right)\right)
$$

holds. Because there is a constant $\gamma>0$ with $\operatorname{xc}\left(P\left(\mathcal{M}_{i}\right)\right) \leq \gamma \cdot\left|E_{i}\right|^{2}$ for each $i=1, \ldots, k$ (recall that each leaf is graphic, cographic or has size bounded by $10)$, and $\sum_{i=1}^{k}\left|E_{i}\right|$ can be bounded linearly in $|E|$ due to Lemma 2, we can further estimate

$$
\sum_{i=1}^{k} \mathrm{xc}\left(P\left(\mathcal{M}_{i}\right)\right) \leq \gamma \cdot \sum_{i=1}^{k}\left|E_{i}\right|^{2} \leq \gamma \cdot\left(\sum_{i=1}^{k}\left|E_{i}\right|\right)^{2}=\mathcal{O}\left(|E|^{2}\right),
$$

which gives the claim.

Suppose we are given some matrix $A \in \mathbb{F}_{2}^{m \times n}$ defining a regular matroid $\mathcal{M}=\mathcal{M}(A)$. It is possible to compute a decomposition tree for $\mathcal{M}$ as in Theorem 1 - including $\mathbb{F}_{2}$-matrices representing all nodes - in time polynomial in $m$ and $n$, see, e.g., [13, Chap. 19-20]. In the next step, for each matrix defining a (co-)graphic leaf node, we compute a graph inducing the same (co-)graphic matroid. This can be also done in polynomial time, see, 
e.g., [16. Since the presented quadratic-size extended formulations for independence polytopes of graphic and cographic matroids can be easily constructed given the underlying graphs, and since the leaf nodes not being graphic or cographic have bounded size, we can thus compute quadratic-size extended formulations for the independence polytopes of all leaf nodes in polynomial time. Together with Lemma 3 and Lemma 4, it is now easy to propagate these extended formulations through the tree until we obtain an extended formulation for $P(\mathcal{M})$. The proof of Theorem 2 shows that this extended formulation has size quadratic in the size of the ground set of $\mathcal{M}$. Thus, a quadratic-size extended formulation for $P(\mathcal{M})$ can be computed in time polynomial in $m$ and $n$.

\section{Concluding Remarks}

A natural question that arises in our context is for which other classes of matroids there are (similar) constructions of polynomial-size extended formulations for the associated independence polytopes. In Section 4, we have seen that whenever a matroid $\mathcal{M}$ can be decomposed by means of 1 -, 2- and 3sums, the extension complexity of $P(\mathcal{M})$ can be bounded by the sum of the extension complexities of the leaf nodes' independence polytopes. However, not many classes of matroids are known that admit decompositions using only 1-, 2- and 3-sums and starting from simple building blocks - as in case of regular matroids. As an obvious generalization of regular matroids, linear matroids over $\mathbb{F}_{2}$ do not seem to have such decompositions.

For any fixed field $\mathbb{F}$, does $\operatorname{xc}(P(\mathcal{M}))$ grow polynomially (in the dimension) for every $\mathbb{F}$-linear matroid $\mathcal{M}$ ?

As mentioned in the introduction, we know from 11 that there exists a family of independence polytopes of matroids whose extension complexities grow exponentially in their dimension. Moreover, following the argumentation in [1], a random sequence of independence polytopes of matroids has this property. However, no such family is known explicitly. In the light of Rothvoss' exponential lower bound on the extension complexities of matching polytopes [12] one might think of matching matroids []. (where, for a given graph $G$, the independent sets are the subsets of nodes that can be covered by some matching of $G$ ) as a candidate family. However, Sam Fiorini recently observed (personal communication) how to construct polynomial-size extended formulations for the independence polytopes of matching matroids by exploiting Edmonds-Galaidecompositions.

Given a candidate matroid $\mathcal{M}$, the question arises, how to prove a nontrivial lower bound on $\mathrm{xc}(P(\mathcal{M}))$. A technique that has been established to provide simple proofs for super-polynomial lower bounds on the extension complexities of many combinatorial polytopes (starting from the correlation polytope, see, e.g., [7), is to prove lower bounds on the rectangle covering number. In terms of the independence polytopes of matroids $\mathcal{M}=(E, \mathcal{I})$ with 
rank function $r$, a rectangle can be defined as a set $\mathcal{F} \times \mathcal{V}$, where $\mathcal{F}$ is a set of non-empty subsets of $E$, and $\mathcal{V}$ is a set of independent sets such that

$$
|I \cap S| \leq r(S)-1
$$

holds for all $(S, I) \in \mathcal{F} \times \mathcal{V}$. Up to an additive term of $\mathcal{O}(|E|)$, the rectangle covering number of $P(\mathcal{M})$ is defined as the smallest number of rectangles needed to cover all pairs $(S, I)$ with $S \subseteq E, I \in \mathcal{I}$ that satisfy (4) and is known to provide a lower bound on the extension complexity of $P(\mathcal{M})$, see 3 . Unfortunately, it turns out that the rectangle-covering number of independence polytopes of matroids cannot provide super-polynomial bounds:

Proposition 2 Given a matroid $\mathcal{M}=(E, \mathcal{I})$, the rectangle-covering number of $P(\mathcal{M})$ is at most $\mathcal{O}\left(|E|^{2}\right)$.

Proof Observe that a pair $(S, I)$ with $S \subseteq E, I \in \mathcal{I}$ satisfies (4) if and only if

- there exists some $e \in S \backslash I$ such that $I \cup\{e\} \in \mathcal{I}$, or

- there exist some $e \in S \backslash I$ and $f \in I \backslash S$ such that $(I \backslash\{f\}) \cup\{e\} \in \mathcal{I}$.

Thus, each such pair is contained in a rectangle of type

$$
\{S \subseteq E \mid e \in S\} \times\{I \in \mathcal{I} \mid e \notin I, I \cup\{e\} \in \mathcal{I}\}
$$

for some $e \in E$, or in a rectangle of type

$$
\{S \subseteq E \mid e \in S, f \notin S\} \times\{I \in \mathcal{I} \mid e \notin I, f \in I,(I \backslash\{f\}) \cup\{e\} \in \mathcal{I}\}
$$

for some $e, f \in E$.

Acknowledgements We would like to thank Klaus Truemper for valuable comments on the decomposition of matroids.

\section{References}

1. Conforti, M., Kaibel, V., Walter, M., Weltge, S.: Subgraph polytopes and independence polytopes of count matroids. Operations Research Letters 43(5), 457-460 (2015)

2. Edmonds, J.: Submodular functions, matroids, and certain polyhedra. Combinatorial structures and their applications pp. 69-87 (1970)

3. Fiorini, S., Kaibel, V., Pashkovich, K., Theis, D.O.: Combinatorial bounds on nonnegative rank and extended formulations. Discrete Math. 313(1), 67-83 (2013). URL http://arxiv.org/abs/1111.0444

4. Fiorini, S., Massar, S., Pokutta, S., Tiwary, H.R., de Wolf, R.: Linear vs. semidefinite extended formulations: exponential separation and strong lower bounds. In: STOC, pp. 95-106 (2012)

5. Grande, F., Sanyal, R.: Theta rank, levelness, and matroid minors. arXiv:1408.1262 (2014)

6. Iwata, S., Kamiyama, N., Katoh, N., Kijima, S., Okamoto, Y.: Extended formulations for sparsity matroids. Mathematical Programming (printed version to appear) (2015). DOI 10.1007/s10107-015-0936-8

7. Kaibel, V., Weltge, S.: A short proof that the extension complexity of the correlation polytope grows exponentially. Discrete \& Computational Geometry 53(2), 396-401 (2015). DOI 10.1007/s00454-014-9655-9. URL http://dx.doi.org/10.1007/s00454-014-9655-9 
8. Lawler, E.L.: Combinatorial optimization: networks and matroids. Courier Corporation (1976)

9. Martin, R.K.: Using separation algorithms to generate mixed integer model reformulations. Oper. Res. Lett. 10(3), 119-128 (1991). DOI http://dx.doi.org/10.1016/0167-6377(91)90028-N. http://www.sciencedirect.com/science/article/pii/016763779190028N

10. Oxley, J.G.: Matroid theory, vol. 1997. Oxford University Press (1992)

11. Rothvoss, T.: Some $0 / 1$ polytopes need exponential size extended formulations. Math. Program. Ser. A pp. 1-14 (2012). DOI 10.1007/s10107-012-0574-3. URL http://dx.doi.org/10.1007/s10107-012-0574-3

12. Rothvoss, T.: The matching polytope has exponential extension complexity. In: Proceedings of the 46th Annual ACM Symposium on Theory of Computing, STOC '14, pp. 263-272. ACM, New York, NY, USA (2014). DOI 10.1145/2591796.2591834. URL http://doi.acm.org/10.1145/2591796.2591834

13. Schrijver, A.: Theory of linear and integer programming. John Wiley \& Sons, Inc., New York, NY, USA (1986)

14. Schrijver, A.: Combinatorial Optimization - Polyhedra and Efficiency. Springer (2003)

15. Seymour, P.D.: Decomposition of regular matroids. Journal of combinatorial Theory, Series B 28(3), 305-359 (1980)

16. Tutte, W.T.: An algorithm for determining whether a given binary matroid is graphic. Proceedings of the American Mathematical Society 11(6), pp. 905-917 (1960). URL http://www.jstor.org/stable/2034435

17. Williams, J.C.: A linear-size zero-one programming model for the minimum spanning tree problem in planar graphs. Networks 39(1), 53-60 (2002). DOI 10.1002/net.10010. URL http://dx.doi.org/10.1002/net.10010 